\title{
O uso de drogas entre adolescentes: Uma revisão integrativa
}

\author{
The use of drugs among adolescents: An integrative review \\ El uso de drogas entre adolescentes: Una revisión integradora
}

Recebido: 08/04/2021 | Revisado: 15/04/2021 | Aceito: 20/04/2021 | Publicado: 04/05/2021

\author{
Maria Isabel Félix da Silva \\ ORCID: https://orcid.org/0000-0002-3245-1928 \\ Universidade Estadual da Paraíba, Brasil \\ E-mail: isabelfelixadpb@gmail.com \\ Leonara Vitória Brito Oliveira \\ ORCID: https://orcid.org/0000-0002-8087- 4183 \\ Universidade Estadual da Paraíba, Brasil \\ E-mail: leonaranz@outlook.com \\ Clésia Oliveira Pachú \\ ORCID: https://orcid.org/0000-0002-7356-6297 \\ Universidade Estadual da Paraíba, Brasil \\ E-mail: clesiapachu@hotmail.com
}

\begin{abstract}
Resumo
A adolescência, fase da vida representada por momentos de descobertas, apresenta-se como emblemática no contexto social do consumo de drogas, mostrando-se cada dia mais crescente. Objetivo: Trata-se de estudo qualitativo descritivo, que objetivou analisar o impacto do uso de drogas psicoativas durante a adolescência, realizado no período de março de 2021, através de revisão integrativa da literatura científica. Metodologia: Realizou-se busca em bases nacionais e internacionais, incluindo a Literatura Latino-Americana e do Caribe em Ciências da Saúde (LILACS), SciELO Brasil e a PubMED, utilizou-se na estratégia de busca os operadores Booleanos e os respectivos Descritores em Ciências da Saúde (DeCS) em português: Drogas-Adolescentes; Substâncias Psicoativas-Adolescentes; Abuso de Substâncias-Adolescentes; Uso de drogas-Adolescentes. Resultados: Obteve-se um retorno de 2122 artigos na base de dados LILACS, 425 na SciELO Brasil e 96 na PubMED, totalizando 2643 artigos, sendo que, na delimitação do critério de inclusão, restaram 35, e apenas 19 artigos foram selecionados para compor o corpus da pesquisa. Constatou-se que quanto mais cedo o início do consumo de substâncias psicoativas, mais visível são os efeitos das drogas nos comportamentos a longo prazo. Além disso, esse consumo abarca também, consequências no desenvolvimento cognitivo do púbere, como, déficit no processo de aprendizagem. Conclusões: Evidenciou-se uma diversidade de fatores vinculados ao uso de drogas na adolescência, dentre os quais, a dinâmica do funcionamento familiar, o contexto cultural, conflitos específicos dessa fase e condição socioeconômica se destacaram como vias de gatilho mais presentes para a inserção dos adolescentes no mundo das drogas.
\end{abstract}

Palavras-chave: Adolescentes; Drogas Psicoativas; Uso de drogas.

\begin{abstract}
The adolescence, stage of life represented by moments of discovery, presents itself as emblematic in the social context of drug use, which represents more and more growing each day. Objective: This is a descriptive qualitative study, which aimed to analyze the impact of using psychoactive drugs during adolescence it was executed in the period of March 2021, through an integrative review of the scientific literature. Methodology: The search was carried out on national and international basis, including the Literatura Latino-Americana e do Caribe em Ciências da Saúde (LILACS), SciELO Brasil and PubMED, the search strategy was to use Boolean operators the following Health Sciences Descriptors (DeCS) in Portuguese: Drugs-Adolescents; Psychoactive Substances-Adolescents; Substance Abuse-Adolescents; Drug useAdolescents. Results: A return of 2122 articles was obtained in the LILACS database, 425 in SciELO Brazil and 96 in PubMED, totaling 2643 articles, and in the delimitation of the inclusion criterion, 35 remained, and only 19 articles were selected to compose the research corpus. It was noticed that the earlier starts the use of psychoactive substances, the more visible are the effects of drugs on long-term behavior. Furthermore, this consumption also includes consequences on the cognitive development of the pubescent, such as a deficit in the learning process. Conclusions: A diversity of factors related to drug use in adolescence were evidenced, among which, the dynamics of family functioning, the cultural context, specific conflicts of this stage of life and socioeconomic condition stood out as the most present trigger pathways for the insertion of adolescents in the world of drugs.
\end{abstract}


Keywords: Adolescents; Psychoactive drugs; Use of drugs.

\begin{abstract}
Resumen
La adolescencia, fase de la vida representada por momentos de descubrimiento, se presenta como emblemática en el contexto social del consumo de drogas, siendo cada día más creciente. Objetivo: Se trata de un estudio cualitativo descriptivo, que objetivó analizar el impacto del uso de las sustancias psicoactivas durante la adolescencia, fue realizado en el período de marzo de 2021, a través de una revisión integradora de la literatura científica. Metodología: Se realizó una búsqueda en bases nacionales e internacionales, incluyendo la Literatura Latino-Americana e do Caribe em Ciências da Saúde (LILACS), SciELO Brasil y PubMED, la estrategia de búsqueda utilizó los operadores booleanos y sus Descriptores en Ciencias de la Salud (DeSC) en portugués: Drogas-Adolescentes; Sustancias Psicoactivas-Adolescentes; Abuso de sustancias-Adolescentes; Uso de drogas-Adolescentes. Resultados: Se obtuvo una devolución de 2122 artículos en la base de datos LILACS, 425 en SciELO Brasil e 96 en PubMED, totalizando 2643 artigos, pero en la delimitación del criterio de exclusión, se mantuvo 35, y solo se seleccionaron 19 artículos para componer el corpus de la investigación. Se encontró que cuanto antes se inicia el uso de sustancias psicoactivas, más visibles son los efectos de las drogas en el comportamiento a largo plazo. Además, este consumo también incluye consecuencias sobre el desarrollo cognitivo del adolescente, como, déficit en el proceso de aprendizaje. Conclusiones: Se evidenció una diversidad de factores relacionados con el uso de drogas en la adolescencia, entre los cuales, la dinámica del funcionamiento familiar, el contexto cultural, los conflictos específicos de esta fase y la condición socioeconómica se destacaron como las vías desencadenantes más presentes para la inserción de los adolescentes en el mundo de las drogas.

Palabras clave: Adolescentes; Drogas psicoactivas; Uso de drogas.
\end{abstract}

\title{
1. Introdução
}

O consumo de drogas durante a fase da adolescência representa um tema recorrente no âmbito da literatura científica. Nesse sentido, a adolescência se apresenta como um período transitório de vivências e de múltiplas adaptações, tanto no sentido subjetivo quanto no social e se compreende um momento atravessado por uma polissemia de faces, que é demarcada pelas interações que o adolescente faz durante seu desenvolvimento (Ferreira \& Nelas, 2016). Nessa perspectiva, ao se debruçar acerca da vivência do uso de drogas psicoativas durante esse período da vida humana, deve-se levar em consideração o contexto em que o adolescente em questão se encontra inserido, bem como, as múltiplas dimensões e complexidades que permeiam o consumo de drogas e compreendê-lo a partir da subjetividade nos aspectos próprios da fase em que se encontram (Paula, Jorge, Lima \& Bezerra, 2017).

Nesse contexto, faz-se importante salientar que o uso de substâncias psicoativas ilícitas ou lícitas, atingem aspectos de ordem psíquica, emocional, cognitiva e social do adolescente, fase biopsicossocial, que por vezes, pode ser vivida de maneira complexa e conflituosa (Nadaleti, Carvalho, de Assis, da Silva, \& Chaves, 2018). Nesse sentido, salienta-se que a adolescência representa um período onde se evidencia um cenário de vulnerabilidade diante das situações ambientais e socioeconômicas (Bittencourt, França, Goldim, 2015).

Assim sendo, ao abordar acerca dos diferentes fatores preditivos, observa-se uma sucessão de influenciadores do comportamento de utilização das drogas entre adolescentes. Evidencia-se a participação da família como um fator de proteção ou indução quanto ao uso das drogas, ocupando a posição de um dos grupos que mais impactam no desenvolvimento de adicção (Vázquez, Oviedo \& Olalde, 2018). Ademais, aspectos como a diversidade cultural e demográfica do país, bem como, a questão de gênero têm colaborado com o consumo de drogas psicoativas na adolescência (Sousa, Stelko-Pereira, Chaves, Moreira, Santos \& Pillon, 2017; Pereira, Resende, Campos, Duarte, Cavalcante \& Machado, 2015). Além de, baixa escolaridade, inexistência de práticas religiosas, aspectos raciais, também se configuram como contextos de destaque que influenciam o consumo de drogas (Santos, Mota, da Silva Carvalho, de Araujo, Gomes \& de Oliveira, 2017). 
A partir dessa reflexão, compreende-se a necessidade de estudos direcionados a esse público que se encontra, paulatinamente, entrelaçado com a cultura do consumo de drogas. De acordo com Morello, Pérez, Peña, Braun, Kollath-Cattano, Thrasher \& Mejía (2017), ter informações que sejam atuais sobre esse consumo na adolescência e também uma discussão pertinente acerca dos fatores sociais e das personalidades relacionadas e este fenômeno, facilita o desenvolvimento de políticas públicas direcionadas à prevenção do consumo dessas substâncias.

À vista disso, ao notar a necessidade de pesquisas e também o visível acréscimo no consumo de drogas entre os adolescentes, este estudo objetivou analisar o impacto desse uso e as implicações desse comportamento nas múltiplas dinâmicas das vivências dos púberes, analisando portanto, os fatores que induzem o uso de drogas nos primórdios dessa fase específica da vida, por intermédio de uma revisão da literatura.

\section{Metodologia}

O presente estudo qualitativo descritivo, realizado no período de março de 2021, utilizou-se de revisão integrativa de artigos da literatura científica. A análise dos artigos a partir da revisão integrativa proporciona uma sintetização de pesquisas disponíveis acerca de determinado tema e encaminha a prática embasada em conhecimento científico. Assim, este método configura-se como uma Prática Baseada em Evidências (Souza, Silva \& Carvalho, 2010). A coleta de dados foi realizada em bases nacionais e internacionais; Literatura Latino-Americana e do Caribe em Ciências da Saúde (LILACS), SciELO Brasil e a PubMED, identificando e selecionando artigos publicados em revistas científicas (Figura 1).

Posto isto, utilizou-se na estratégia de busca os operadores Booleanos e respectivos Descritores em Ciências da Saúde (DeCS): "Drogas" AND “Adolescentes"/ "Substâncias Psicoativas" AND "Adolescentes"/ "Abuso de Substâncias" AND "Adolescentes"/ "Uso de drogas" AND "Adolescentes".

Dessa maneira, os critérios de inclusão adotados para a amostra deste estudo foram: artigos que estabelecem como população os adolescentes, estudos qualitativos ou quantitativos, ter sido publicado entre 2015 e 2020, artigos originais; na língua portuguesa, inglesa ou espanhola; com abordagens qualitativas com acesso livre on-line, com texto na íntegra. Já os critérios de exclusão foram: artigos que constituem como população as crianças, adultos e idosos; resultados de pesquisas direcionados a temáticas fora do eixo da pesquisa: sexualidade, desempenho ocupacional, atuação de profissionais frente a problemática, comportamento violento e infração. 
Figura 1- Representação esquemática da seleção e inclusão de artigos.

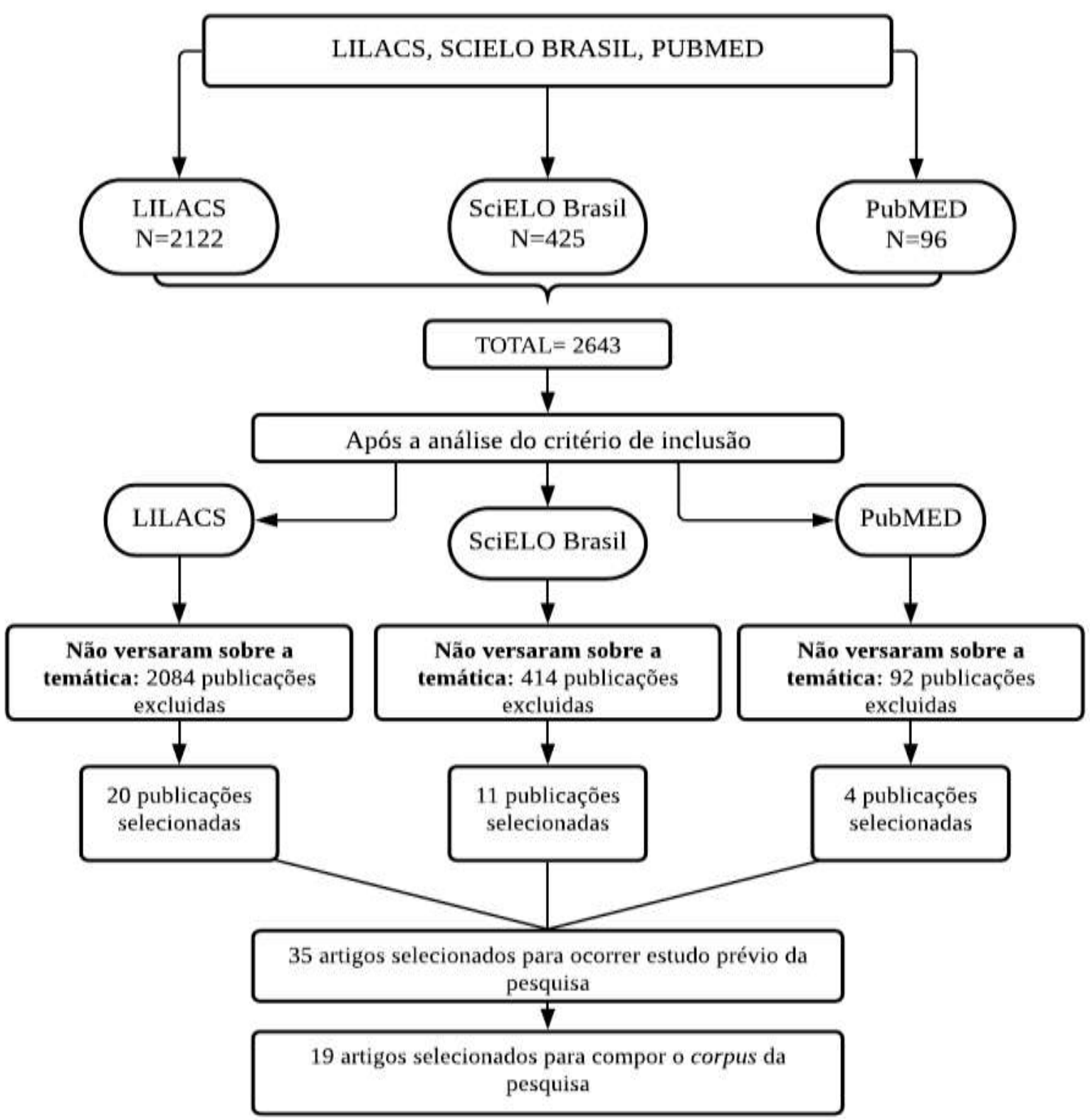

Fonte: Os autores (2021).

Foram identificados 2122 artigos na base de dados LILACS, 425 na SciELO Brasil e 96 na PubMED, totalizando 2643 artigos. Do material selecionado na delimitação posta pelo critério de inclusão, restaram 35 artigos prévios para leitura. Tendo em vista, os artigos que não estavam condizentes com a linha da pesquisa, outros que não estavam disponibilizados de maneira online ou que não foram encontrados, utilizou-se no corpus deste estudo, apenas 19 artigos que foram considerados mais relevantes ante a temática abordada. 


\section{Resultados e Discussão}

A adolescência representa um momento de grande impacto no desenvolvimento humano, moldando-se como período crucial no que concerne ao uso de drogas. De acordo com o Ministério da Saúde, a adolescência corresponde à faixa etária entre 10 a 19 anos - conforme critério definido pela Organização Mundial da Saúde (OMS) - e consiste em um complexo processo de crescimento e desenvolvimento biopsicossocial (Brasil, 2005). Desse modo, em conformidade com o autor supramencionado, a noção de adolescência que permeia na sociedade remete a uma fase de rebeldia, desordem, irresponsabilidade e está intrinsecamente associado com comportamento de risco, dentre estes, o consumo de drogas lícitas e ilícitas.

Nessa perspectiva, ao explorar a literatura científica que aborda acerca do consumo de drogas nessa faixa etária, verificouse que os estudos em sua maioria evidenciam um contexto de violência, inexistência de afeto e desequilíbrio familiar em que o adolescente se encontra inserido. Foi possível observar, com base em Vázquez et al. (2018), que o funcionamento familiar pode refletir sobre o uso de drogas dos membros, pois, os adolescentes desta pesquisa relataram que a opressão diária, a violência, a falta de afeto e a moralidade nesse contexto de família, são reflexos dos comportamentos inadequados dos familiares perante ao consumo de drogas, a família portanto, funciona como fator protetivo de promoção a resiliência, ou como fator de indução ao risco do consumo.

Além disso, Vázquez et al. (2018) ainda acrescentam que diante de uma atuação familiar deficitária, o sofrimento do adolescente tende a refletir na adicção como uma maneira de fugir da realidade ao qual vivencia. Nesse sentido, constatou-se que o adolescente experimenta uma crise existencial durante o consumo de drogas que lhe permite sentir sensações, desconectando-lhe das preocupações de si mesmo por sua existência.

Análises acerca dos fatores implicados na entrada dos adolescentes ao universo das drogas exprimem uma série de fatores individuais e ambientais como responsáveis por esse fenômeno: vulnerabilidade socioeconômica, disponibilidade da droga e características do funcionamento familiar. Ademais, os estudos desvelam risco avultado se o adolescente habitar em um contexto de elevados níveis de conflito, deficiência na comunicação com os pais, progenitores e amigos usuários de substâncias psicoativas, estilo parental negligente e permissivo (Peuker, Caovilla, Costa, Crístofer \& Mosmann, 2020).

Um estudo realizado por Peuker et al. (2020) com adolescentes de 12 anos, identificou que o consumo de drogas nessa fase, se deve a conflitos com os pais. Desse modo, essa constatação sugere que o conflito no exercício da parentalidade e o envolvimento dos filhos no conflito parental repercutem negativamente no adolescente. Portanto, faz-se imprescindível destacar a importância do âmbito familiar na dinâmica do uso de drogas entre adolescentes. Nesse sentido, comportamentos parentais que mostram pouca responsabilidade por seus filhos, como a inabilidade dos pais de compreenderem os problemas dos filhos, estão diretamente relacionados ao uso de drogas ilícitas, como foi evidente nos adolescentes que participaram da Pesquisa Nacional de Saúde Escolar, executada no ano de 2015 (Antunes, Rivadeneira-Guerrero, Goulart \& Oenning, 2018).

É válido salientar que a necessidade de políticas públicas direcionadas para um público com idade entre 10-19 anos, promoverá a prevenção ao uso precoce de drogas psicoativas. Nesse sentido, em estudo executado com adolescentes brasileiros que iniciaram a adolescência recentemente, foi possível constatar uma padronização de comportamentos associados a uma série de fatores de riscos relacionados ao consumo de álcool: baixo desempenho escolar, agressividade, transtorno de álcool de um membro familiar e envolvimento com outras drogas (Conegundes, Valente, Martins, Andreoni \& Sanchez 2020).

Por conseguinte, ao endossar Almeida \& Lana (2020) deixam notório a importância do contexto sociocultural, como fator de impacto no contexto do consumo de substâncias psicoativas. Observou-se que, adolescentes reproduzem comportamentos 
sociais e afirmações constatadas pela comunidade as quais se encontram inseridos. Portanto, ao analisar a dinamicidade do contexto de intervenção e prevenção quanto ao consumo de drogas, deve-se considerar ações direcionadas tanto no espaço escolar quanto no familiar.

Corrobora com essa acepção Morello et al. (2017) ao postular, por meio de um estudo realizado em três cidades argentinas, que o suporte parental representa um dos fatores de risco mais importantes, no que concerne a utilização de substâncias psicoativas por adolescentes. Acrescentaram que, ter amigos que fumam ou bebem e uma procura por fortes emoções, também se configuraram como fatores de risco para o uso de drogas.

Nessa linha de raciocínio, uma outra pesquisa realizada por Raposo, Costa, Valença, Zarzar, Diniz, Colares \& Franca (2017) com adolescentes, demonstrou na pesquisa que a prevalência do uso de drogas ilícitas no contexto dos participantes foi elevada quando comparada com outros estudos que foram realizados no Brasil. Evidenciando além disso que, o consumo excessivo de álcool foi a variável que mais influenciou o uso de maconha, cocaína e inalantes. Assim sendo, em virtude das limitações, por ser um estudo transversal, não aborda, o efeito preditor sobre o uso dessas drogas.

Buscando identificar os fatores preditivos que contribuem para a inserção do adolescente no mundo das drogas, Marin, Peuker \& Kessler (2019), enfatizaram que, por ainda estarem vivenciando um processo de desenvolvimento biológico, emocional, comportamental e social, o consumo de drogas como o álcool, resulta em consequências negativas ao cérebro, comprometendo as regiões corticais e subcorticais.

Desse modo, os autores também sugerem que essas alterações cerebrais podem conduzir ao comprometimento de vários processos cognitivos, afetivos e comportamentais, a exemplo da aprendizagem e tomada de decisão. Além disso, as vias do sistema límbico que afetam adversamente os aspectos comportamentais e emocionais do desenvolvimento podem ser alteradas. Assim, o uso de álcool na adolescência tem sido correlacionado com dificuldades de aprendizagem, falta de motivação, absenteísmo e abandono escolar Marin et al., 2019).

Corrobora com essa linha de raciocínio, o estudo realizado por Soares, Farias \& Monteiro (2019), associando a prevalência do consumo de drogas entre adolescentes e o absenteísmo escolar. Nesse sentido, a ausência dos estudantes durante um período de tempo na escola e verificando os padrões de consumo de drogas, mostraram-se significativas para aqueles que consumiam periodicamente o álcool, tabaco e inalante de maconha de maneira pesada. Nessa perspectiva, estudantes que consumiam substâncias psicoativas, quando questionados em outra pesquisa acerca da qualidade de vida afirmaram em linhas gerais que o não uso dessas substâncias está interligado com melhores níveis de satisfação com a saúde e, também, com uma boa qualidade de vida (Gonçalves, Wernet, Costa, Silva Júnior, Moura \& Pillon, 2020). Isto reafirma a necessidade de políticas de prevenção ao uso de drogas cada vez direcionada a esse público.

Ademais, constatou-se com base em Marin et al. (2019), que problemas de ordem emocional, precisamente indicativos de ansiedade e depressão, favorecem a ingestão de álcool por adolescentes. Assim, foi possível constatar uma infinidade de fatores predisponentes ao uso de substâncias psicoativas por adolescentes que demarcam a continuidade desse consumo também na vida adulta.

No estudo de Boarini (2018), foi possível perceber a vulnerabilidade do segmento juvenil em condição financeira instável perante o assédio do tráfico de drogas. Contudo, a autora propõe que nem todos os adolescentes implicados com o uso/abuso das drogas integram a classe social desfavorecida economicamente. Nesse sentido, estudos atestam que alunos da rede de educação privada de todas as capitais do país têm maior percentual de consumo de drogas em relação aos discentes da rede pública de ensino. 
Ainda, no mesmo estudo, a autora destaca a crença existente de que a inserção da mulher ao mercado de trabalho motivou o interesse dos filhos pelas drogas. Nesse contexto, evidenciou-se uma exigência da necessidade da presença materna na educação dos filhos como sendo indispensável para o funcionamento familiar. Enquanto, a ausência da figura paterna se encontra naturalizada sob a justificativa de que foi imputado a mulher a incumbência de preparar os seus membros para adaptar-se a sociedade (Boarini, 2018).

Nesse sentido, Nadaleti et al. (2018), na tentativa de avaliar os problemas associados ao uso de álcool entre adolescentes, constataram a implicação da condição socioeconômica em relação ao consumo precoce. Observaram ainda que, não houve distinção de utilização entre meninos e meninas. Os autores também propõem que os adolescentes dispõem de conhecimentos quanto aos problemas suscitados pelo uso/abuso de substâncias psicoativas nessa fase da vida. À vista disso, pôde-se notar controvérsias em referência a outros estudos.

Outros achados pertinentes, no mesmo estudo, foi o fato das variáveis familiares serem apontadas como fator de proteção, diminuindo os riscos do adolescente se envolver com drogas lícitas e/ou ilícitas, quando há uma comunicação não violenta entre pais e filhos (Nadaleti et al. 2018). Essa informação vai ao encontro de outras pesquisas, que relacionam a percepção de apoio familiar e a comunicação com os pais como indicativos de menor consumo.

Dessas acepções pode-se ressaltar, com base em Centurión \& Rodríguez (2017), a existência de pesquisas suficientes para sustentar que os adolescentes são mais vulneráveis ao uso de substâncias lícitas/ilícitas. Isto se deve ao fato de sua identidade ainda estar em processo de construção, além de que, ele enfrenta constante oscilação de emoções que oportuniza a experimentação. Em consequência, quanto maior a precocidade de início do consumo, maior os efeitos desse comportamento a longo prazo.

Corroborando com as constatações dos autores, anteriormente mencionados, pode-se inferir que os danos decorrentes do uso de substâncias psicoativas nessa fase da vida afetam a relação familiar, promovem o isolamento social e contribuem para o desenvolvimento de distúrbios mentais (Sousa et al., 2017). Essas colocações refletem o contexto de vulnerabilidade psicossocial que vivencia o adolescente.

Partindo dessa perspectiva, Sousa et al. (2017), concordam que os índices de prevalência de uso de drogas se deve a diversidade cultural e demográfica do país, bem como as mudanças comportamentais vivenciadas pelos adolescentes. Ainda com base nesse autor, observou-se que o consumo também se encontra influenciado pela variável religião. Segundo os autores, adolescentes que professam fé em segmentos religiosos que adotam normas tradicionalistas, funcionam como fator de prevenção. Nesse sentido, ao se inserirem nesses contextos, os adolescentes se conectam com valores, símbolos, comportamentos e práticas sociais que favorecem o resguardo quanto ao uso de drogas. O estudo proposto pelos autores retromencionados, atentou ainda para associação entre uso de drogas e transtorno psiquiátrico na adolescência, pelo fato de as drogas alterarem o estado de consciência, deixando-o propenso a manifestar afecções psíquicas. Entretanto, deve-se considerar que essa correlação pode ser uma tentativa do adolescente amenizar o sofrimento psíquico quando exposto a tensão promovida pelo contexto social.

Ao estudar os fatores associados à iniciação tabágica em adolescentes escolares, Teixeira, Guimarães \& Echer (2017), verificaram uma compatibilidade com a variável cor. Fato este, segundo os autores, deve-se a condição sociocultural de marginalização, exclusão, e situação econômica precária de negros e pardos. Diante disso, notou-se que a literatura científica não dispõe de muitos recursos que correlacione o uso de drogas com a cor dos indivíduos pelo fato de em outros estudos não ter tido relevância estatística. O mesmo estudo evidenciou que famílias com elevado poder aquisitivo apresentam maior risco do adolescente ser tabagista quando comparados com famílias em situação financeira precária. Entretanto, observa -se que o consumo de drogas não é uma condição própria de famílias em situação de vulnerabilidade socioeconômica. 
Nesse sentido, ratifica Santos et al. (2017), partindo da investigação acerca dos fatores associados ao consumo de drogas em idade precoce que existe elevada associação estatística entre baixa escolaridade, falta de prática religiosa e maior prevalência de consumo em adolescentes do sexo masculino, pertencentes à raça negra. Para as autoras, esse evento antecipado pode resultar em transtornos psiquiátricos, bem como, potencializar conflitos familiares, atitudes violentas, abandono ou dificuldades no contexto escolar. Diante desse encadeamento de consequências, observa -se que os dados corroboram com outros estudos vinculados à adolescência em contexto de drogadição.

Mediante a situação, Pereira et al. (2015), inferem, a partir de levantamentos com adolescentes acerca do uso de substâncias psicotrópicas que a prevalência de uso se encontra frequente entre o sexo masculino. Embora não seja regra, essa constatação se apresenta como indicativo que os estudos envolvendo a temática, aqui descrita, devem contemplar a discussão de gênero. Os autores apontam também, que isso se dá devido à cultura machista que permeia o âmbito social, estimulando o menino a comprovar sua coragem, o conduzindo a adotar tal comportamento a fim de se inserir em determinado contexto. Por outro lado, a menina tende a agir diferente pelo fato de ser vista como frágil e suscitar maior proteção. Embora haja indícios de veracidade nessas colocações, salienta-se que, o consumo de drogas nessa faixa etária não é exclusividade do sexo masculino e nem tampouco, a menina está isenta desse comportamento.

Observando outras evidências para o uso de substâncias psicoativas por adolescentes, o estudo de Pereira et al. (2015), infere que a principal motivação do adolescente ao iniciar o uso de drogas está relacionada à curiosidade acerca dos efeitos que a droga pode conferir. O mesmo estudo, encontrou respaldo para certificar que, embora haja divergências concomitantes, as variáveis raciais são apontadas como fator predisponentes ao uso de drogas. Essas observações sugerem que as questões raciais estão ainda muito ligadas à herança cultural escravocrata e segregadora que permeou o processo de colonização do país.

Ainda, com base em Pereira et al. (2015), observou-se que no que corresponde a família, as constituições monoparentais favorecem o envolvimento dos filhos com substâncias psicoativas. Isso se deve ao fato da família, detentora da função de inserir seus membros na sociedade e ser instituidora das relações primárias, influenciar na forma como o adolescente reage frente a ampla oferta de drogas hodiernamente. Diante desse contexto, ao se pensar na problemática da adolescência e o uso de drogas, compactua-se do princípio que o jovem está em busca de um suporte para aliviar as mais diversas fontes de tensão próprias da fase que vivenciam.

\section{Considerações Finais}

Ao entender os diferentes contextos implicados nas múltiplas dimensões da adolescência, compreende-se, ao analisar a utilização de drogas nessa fase, um período de descoberta, a correlação da falta de informação de qualidade, o incentivo social para o consumo e o contexto social que este sujeito se encontra inserido, além do âmbito familiar diretamente ligados com o uso de substâncias psicoativas. Evidencia-se assim, a necessidade da implementação de fatores de proteção nesse período, vislumbrando a indispensabilidade de uma atenção especializada para uma fase que por vezes se apresenta negligenciada, principalmente no que concerne à esfera institucional familiar.

Constatou-se que, quanto mais cedo o início do consumo de substâncias psicoativas entre adolescentes, mais evidente se torna os efeitos das drogas nos comportamentos a longo prazo. Assim, a utilização das drogas traz diversas consequências no desenvolvimento cognitivo do púbere. Nesse sentido, o uso dessas substâncias traz diversas consequências no tocante ao contex to 
de aprendizagem do adolescente, como o absentismo, abandono e fracasso no ambiente escolar. Outrossim, o isolamento social e o desenvolvimento de distúrbio mental também se configuram como resultado da utilização da droga.

Ademais, verificou-se que os fatores que conduzem ao uso de drogas psicoativas são a vulnerabilidade socioeconômica, disponibilidade para acesso às drogas, desejo de fugir da realidade, amigos que fumam ou que bebem, fortes emoções para ter novas experiências e a curiosidade para provar determinada substância. Além das características no núcleo familiar, tipos de relações parentais são consideradas como vias de gatilho para a entrada do adolescente no mundo das drogas. Conquanto, aspectos como seguir determinada religião, diálogo no ambiente familiar, políticas de prevenção direcionadas para este público, se configuram como condições de fatores de prevenção para utilização de substâncias psicoativas.

Sendo assim, as políticas de prevenção não devem focar na droga, mas sim, na necessidade de cada sujeito, incluindo a família numa rede de suporte, pois compreende -se que, para atuar como fator de proteção frente ao uso de drogas por seus membros, essa família precisa dispor de condições prévias para tal. Além disso, vê-se como de fundamental importância reconhecer os fatores subjetivos e socioculturais, bem como, a singularidade e as experiências de vida associados ao consumo de drogas por esses indivíduos.

Ressalta-se a necessidade de outros estudos que possam desvelar e compreender o impacto do uso de substâncias psicoativas na vida dos adolescentes e também os fatores desencadeadores dessa problemática social, tendo em vista, a melhoria de possíveis intervenções no contexto de drogadição e também pensar em estratégias possíveis de serem construídas pelos profissionais do Sistema Único de Saúde, para que aja promoção de saúde e prevenção dos prejuízos que a problemática das drogas gera no adolescente e sua família.

\section{Referências}

Almeida, C. S. D., \& Lana, F. C. F. (2020). Relations between sociocultural spaces and the consumption of psychoactive substances by adolescents. Revista gaúcha de enfermagem, 41 .

Antunes, H. D. A., Rivadeneira-Guerrero, M. F., Goulart, B. N. G. D., \& Oenning, N. S. X. (2018). Familiar factors and illicit drug use among Brazilian adolescents: an analysis of the Brazilian National Survey of School Health (PeNSE, 2015). Cadernos de saude publica, 34 , e00009518.

Bittencourt, A. L. P., Garcia, L. F., \& Goldim, J. R. (2015). Adolescência vulnerável: fatores biopsicossociais relacionados ao uso de drogas. Revista Bioética, 23(2), 311-319.

Boarini, M. L. (2018). Drogas na adolescência: desafio à saúde e à educação. Psicologia em Pesquisa, 12(2), 57-67.

Brasil, Ministério da Saúde. (2005). Área de Saúde do Adolescente e do Jovem. Marco legal: saúde, um direito dos adolescentes, Brasília: (editora do Ministério da Saúde), 7 - 9. http://www.saude.sp.gov.br/resources/ses/perfil/profissional-da-saude/grupo-tecnico-de-acoes-estrategicas-gtae/saude-das-populacoes-privadas-deliberdade/saude-dos-adolescentes-em-conflito-com-a-lei/seminario-ministerio-da-saude-fundacao-casa-e-ses/marco_legal_saude_um_direito_de_adolescentes.pdf.

Centurión-Viveros, C. C., \& Rodríguez-Riveros, M. I. (2017). Conocimiento y consumo de drogas ilegales en estudiantes de un colegio público. Mem. Inst. Investig. Cienc. Salud. 2017; 15(2): 14-20.

Conegundes, L. S. O., Valente, J. Y., Martins, C. B., Andreoni, S., \& Sanchez, Z. M. (2020). Binge drinking and frequent or heavy drinking among adolescents: prevalence and associated factors. Jornal de pediatria, 96(2), 193-201.

Ferreira, M., \& Nelas, P. B. (2016). Adolescências... Adolescentes... Millenium-Journal of Education, Technologies, and Health, (32), 141-162.

Gonçalves, A. M. D. S., Wernet, M., Costa, C. D. S. C. D., Silva Júnior, F. J. G. D., Moura, A. A. M. D., \& Pillon, S. C. (2020). Uso de alcohol, tabaco y marihuana: repercusiones en la calidad de vida de los estudiantes. scola Anna Nery, 24(2), e20190284.

Marin, A. H., Peuker, A. C., \& Kessler, F. H. P. (2019). Sociodemographic characteristics, school performance, pattern of consumption and emotional health as risk factors for alcohol use among adolescents. Trends in Psychology, 27(1), 279-292.

Morello, P., Pérez, A., Peña, L., Braun, S. N., Kollath-Cattano, C., Thrasher, J. F. \& Mejía, R. (2017). Factores de riesgo asociados al consumo de tabaco, alcohol y otras drogas en adolescentes escolarizados de tres ciudades de Argentina. Arch. argent. pediatr, 115(2):155-168. 
Research, Society and Development, v. 10, n. 5, e22110514778, 2021

(CC BY 4.0) | ISSN 2525-3409 | DOI: http://dx.doi.org/10.33448/rsd-v10i5.14778

Nadaleti, N. P., Muro, E. S., de Carvalho, C. C., de Assis, B. B., da Silva, D. M., \& Chaves, E. D. C. L. (2018). Avaliação do consumo de álcool entre adolescentes e os problemas associados. SMAD Revista Eletrônica Saúde Mental Álcool e Drogas (Edição em Português), 14(3), 168-176.

Paula, M. L. D., Jorge, M. S. B., Lima, L. L. D., \& Bezerra, I. C. (2017). Experiências de adolescentes em uso de crack e seus familiares com a atenção psicossocial e institucionalização. Ciência \& Saúde Coletiva, 22, 2735-2744.

Pereira, B. M., Resende, K. A., Campos, C. G., Duarte, S. J. H., Cavalcante, R. B., \& Machado, R. M. (2015). Uso de drogas psicotrópicas por adolescentes de escolas públicas. Cogitare Enfermagem, 20(4), 750-757.

Peuker, A. C. W., Caovilla, J. D., Costa, C. B. D., \& Mosmann, C. P. (2020). Uso de alcohol y otras drogas por adolescentes: asociaciones con problemas emocionales y comportamentales y el funcionamiento familiar. Psicologia Clínica, 32(2), 315-334.

Raposo, J. C. D. S., Costa, A. C. D. Q., Valença, P. A. D. M., Zarzar, P. M., Diniz, A. D. S., Colares, V., \& Franca, C. D. (2017). Binge drinking and illicit drug use among adolescent students. Revista de saude publica, 51,83 .

Santos, M. M., Mota, R. S., da Silva Carvalho, M. R., de Araujo, G. S., Gomes, N. P., \& de Oliveira, J. F. (2017). Drug use and associated factors: a cross-sectional study with elementary school adolescents. Online Brazilian Journal of Nursing, 16(1), 64-72.

Soares, F. R. R., Farias, B. R. F. D., \& Monteiro, A. R. M. (2019). Consumption of alcohol and drugs and school absenteeism among high school students of public schools. Revista brasileira de enfermagem, 72(6), 1692-1698.

Sousa, B. de O. P., Stelko-Pereira, A. C., Chaves, Érika de C. L., Moreira, D. da S., Santos, M. A. dos, \& Pillon, S. C. (2017). Drug use and risk factors among school adolescents. Acta Scientiarum. Health Sciences, 39(2), 233-240.

Souza, M. T. D., Silva, M. D. D., \& Carvalho, R. D. (2010). Revisão integrativa: o que é e como fazer. Einstein (São Paulo), 8(1), $102-106$.

Teixeira, C. D. C., Guimarães, L. S. P., \& Echer, I. C. (2017). Fatores associados à iniciação tabágica em adolescentes escolares. Revista Gaúcha de Enfermagem, $38(1), \mathrm{e} 69077$.

Vázquez, M. D. J. P., Oviedo, A. D., \& Olalde, M. G. C. (2018). Life experience of adolescent drug users. Revista da Escola de Enfermagem da USP, 52, e03349e03349. 